\title{
SÍNDROME DE STEVENS-JOHNSON E NECRÓLISE EPIDÉRMICA TÓXICA: RELATO DE CASO
}

\author{
Maria Cristina Almeida de SOUZA ${ }^{1 *}$, Ana Paula dos Santos CORREIA ${ }^{1}$, Gefson Couto MAGRANI ${ }^{1}$, \\ João Carlos de Souza CÔRTES JUNIOR ${ }^{1,2}$ \& Paula Pitta de Resende CÔRTES ${ }^{1,2}$
}

1 Universidade Severino Sombra. Discente do Curso de Medicina. Vassouras, Rio de Janeiro, Brasil.

2 Universidade Federal do Estado do Rio de Janeiro. Rio de Janeiro, Brasil.

*Autor para correspondência: mcas.souza@uol.com.br

DOI: http://dx.doi.org/10.18571/acbm.168

\section{RESUMO}

A Síndrome de Stevens-Johnson (SSJ) e a Necrólise Epidérmica Tóxica (NET) são reações mucocutâneas agudas, caracterizadas por máculas eritematosas, localizadas principalmente no tronco e em membros proximais, que evoluem progressivamente para bolhas flácidas confluentes, levando ao destacamento epidérmico. As drogas são os principais agentes etiológicos. As complicações mais frequentes são a sepse, a ceratoconjuntivite, a hiper ou hipopigmentação da pele e as complicações oculares. Numerosos casos necessitam de internação em unidades de terapia intensiva. Manifestações clínicas da SSJ e da NET são descritas por meio de relato de caso de uma paciente atendida na Unidade de Pronto Atendimento (UPA), diagnosticada com Síndrome de Stevens Johnson, que posteriormente evoluiu para NET.

Palavras-Chave: Medicina Intensiva; Síndrome de Stevens Johnson; Necrólise Epidérmica Tóxica.

\begin{abstract}
Stevens-johnson's syndrome and toxic epidermal necrolysis: case report. Stevens-Johnson Syndrome (SJS) and Toxic Epidermal Necrolysis (TEN) are acute mucocutaneous reactions, characterized by erythematous macules, located mainly in the trunk and proximal limbs, progressively progressing to confluent flaccid blisters, leading to epidermal detachment . Drugs are the main etiological agents. The most frequent complications are sepsis, keratoconjunctivitis, hyper or hypopigmentation of the skin and ocular complications. Numerous cases require hospitalization in intensive care units. Clinical manifestations of SSJ and TEN are described by means of a case report of a patient attended at the Emergency Care Unit, diagnosed with Stevens Johnson Syndrome, who later progressed to NET.
\end{abstract}

Keywords: Intensive Care Medicine; Stevens Johnson Syndrome; Toxical Epidermal Necrolisys.

\section{Introdução}

A Síndrome de Stevens-Johnson (SSJ) foi descrita em 1922 por médicos pediatras norteamericanos ao relatarem o caso clínico de duas crianças que apresentaram febre, estomatite erosiva, acometimento ocular grave e lesões cutâneas disseminadas, descritas como máculas eritematosas, algumas possuindo centro necrótico (FITZPATRICK, 2011; LUPI et al., 2012; RAMOS E SILVA et al., 2010).

Em 1956, Lyell relatou alguns casos de pacientes com perda epidérmica secundária à necrose e criou o termo Necrólise Epidérmica Tóxica (NET). A NET e a SSJ são reações mucocutâneas agudas potencialmente fatais, caracterizadas por máculas eritematosas, localizadas principalmente no tronco e em membros proximais, que evoluem progressivamente para bolhas 
flácidas confluentes, levando ao destacamento epidérmico. São variantes de um único processo, onde a NET é a forma mais grave. Em mais de 50\% dos casos de SSJ e de $80 \%$ a $95 \%$ dos casos de NET, as drogas são os principais agentes etiológicos. Mais de 100 medicamentos de variadas classes farmacológicas estão associados à etiologia da NET e da SSJ. Os mais frequentemente relacionados pertencem ao grupo das sulfonamidas, os anticonvulsivantes, os anti-inflamatórios não hormonais, o alopurinol e a nevirapina. Nos países em que a dipirona é comercializada, essa droga aparece se correlacionando constantemente com as reações. Em 25\% dos casos, nenhum agente etiológico consegue ser identificado, ocasionado à SSJ-NET idiopática (AZULAY, 2013; BULISANI et al., 2006; CEDEÑO et al., 2014; RIVITTI, 2014).

Outras causas associadas a essas doenças são as infecções causadas pelos vírus Herpes simplex, Mycoplasma pneumoniae, Mycobacterium tuberculosis, Streptococcus grupo A, vírus da hepatite B, vírus de Epstein-Barr, Francisella tularensis, Yersinia, Histoplasma, Enterovírus e vírus da Imunodeficiência Humana (HIV), além de neoplasias como a leucemia e os linfomas (FERNÁNDEZ et al., 2011).

Estima-se que a incidência de SSJ e NET seja de 1 a 6 casos por milhão de pessoas/ano e de 0,4-1,2 casos por milhão de pessoas/ano, respectivamente. Essas alterações afetam ambos os gêneros e acometem todas as raças. Embora de baixa prevalência, a mortalidade é de 5\% para SSJ e pode chegar a 40\% para NET, devido à severidade (CEDEÑO, et al., 2014; FITZPATRICK, 2011).

As complicações mais frequentes da SSJ e da NET são a sepse - que quando não reconhecida e tratada, pode levar ao óbito - e a ceratoconjuntivite, que pode acometer a visão e resultar em retração conjuntival, cicatrização e lesão da córnea. Na fase tardia da NET, são citadas como sequelas mais comuns a hiper ou hipopigmentação da pele $(62,5 \%)$, as distrofias das unhas $(37,5 \%)$ e as complicações oculares, dentre as quais se destacam olhos secos, perda da visão e ulceração da córnea. Cicatrizes hipertróficas têm baixa prevalência. $\mathrm{Na}$ fase aguda da doença as complicações que acometem as mucosas, ocorrem em $73 \%$ dos pacientes. Acometimento respiratório é frequente e preocupante. Edema intersticial pode ser de início subclínico, evoluindo para quadro clínico aparente, onde 10-20\% dos pacientes necessitam de ventilação artificial. Todas as complicações e sequelas necessitam ser observadas muito atentamente pela equipe de saúde. Numerosos casos necessitam de internação em unidades de terapia intensiva com fluidos intravenosos, suporte nutricional, roupas leves, camas especiais, analgesia e cateterismo vesical. Os antibióticos são muito importantes nas infecções bacterianas secundárias que, eventualmente, podem levar a morte (EMERICK, et al., 2014; FERNÁNDEZ et al., 2011).

Objetiva-se descrever a evolução das manifestações clínicas da SSJ e da NET por meio de relato de caso de uma paciente atendida na Unidade de Pronto Atendimento (UPA) do município de Três Rios, no sul do Estado do Rio de Janeiro, diagnosticada com Síndrome de Stevens Johnson, que posteriormente evoluiu para NET.

\section{Relato de caso}

J.F.S, gênero feminino, 69 anos, cor negra, histórico de Acidente Vascular Encefálico (AVE) isquêmico sem sequelas ocorrido há 3 anos. Na anamnese, relatou ser hipertensa e fazer uso de losartana, hidroclorotiazida e anlodipino, sem histórico de etilismo e tabagismo.

Transferida de uma Unidade de Saúde, deu entrada na emergência da Unidade de Pronto Atendimento (UPA) do município de Três Rios-RJ, em, com lesões máculas-eritematosas em face, pescoço, tronco, membros superiores e dorso (Figuras 1A, 1B e 1C), além de acometimento de mucosa ocular e oral (Figuras 2A, 2B e 2C). Relatou ter feito uso dos antibióticos ceftriaxona, azitromicina, amoxicilina e do antiinflamatório nimesulida duas semanas anteriores para tratamento de amigdalite e que, quatro dias anteriores ao primeiro atendimento na UPA, quando estava febril, fez uso do analgésico dipirona de 500 miligramas, 1 comprimido de 6 em 6 horas, via 


\section{Biomedica Brasiliensia}

oral. Dois dias após o início do uso da dipirona, iniciou-se um quadro de edema em face, com lesões erosivas nos lábios, que se agravaram dia seguinte, estendendo-se para toda a face. A paciente procurou então atendimento em uma unidade de saúde e, dada a gravidade do caso, foi transferida para a UPA do município de Três Rios, quando foi internada e alojada em quarto de isolamento.

Ao exame clínico, a equipe de saúde da UPA constatou que a paciente apresentava-se lúcida e orientada em tempo e espaço, hidratada, com os seguintes sinais vitais: Pressão Arterial (PA) 110 X 60 mmHg, Frequência Cardíaca (FC) 111 bpm, Frequência Respiratória (FR) 22 irpm, Temperatura Corporal (Tax) $38^{\circ} \mathrm{C}, \quad \mathrm{SatO}_{2} 92 \%$ (em ar ambiente), Murmúrio Vesicular Universalmente Audível (MVUA) sem ruídos adventícios. A pele apresentava lesões em alvo, atípicas, planas e observaram-se lesões crostosas em face, pescoço, tronco, membros superiores, abdômen e dorso, com destacamento de pele em face e pescoço, bem como acometimento de mucosa oral e ocular (Figuras 2A, 2B e 2C). Os dados semiológicos revelaram quadro compatível com Síndrome de Stevens-Johnson, que constituiu a principal hipótese diagnóstica. Paciente alegou nunca ter apresentado quadro parecido e negou histórico de reação alérgica.

Foi suspenso o uso da dipirona - pois se suspeitou que pudesse ser o medicamento o causador da SSJ - bem como o uso dos anti hipertensivos. Iniciou-se então, o tratamento com a prescrição sistêmica de Prednisona $80 \mathrm{mg} /$ dia via oral. Para aplicação nas lesões, foi prescrito uso tópico de sulfadiazina de prata $10 \mathrm{mg} / \mathrm{g}$ associada à dexametasona $1 \mathrm{mg} / \mathrm{g}$, aplicada duas vezes ao dia. Foram solicitados exames laboratoriais complementares, cujos resultados são demonstrados no Quadro 1.

Quadro 1: Resultado de exames laboratoriais (26/10/2016).

\begin{tabular}{|l|l|l|}
\hline Exames & Resultados & Valor de Referência \\
\hline Ureia & $39,8 \mathrm{mg} / \mathrm{dl}$ & De 10,0 a $50,0 \mathrm{mg} / \mathrm{dl}$ \\
\hline Creatinina & $0,77 \mathrm{mg} / \mathrm{dl}$ & De 0,4 a $1,4 \mathrm{mg} / \mathrm{dl}$ \\
\hline Sódio & $126,0 \mathrm{mEq} / \mathrm{L}$ & De 133,0 a $148,0 \mathrm{mEq} / \mathrm{L}$ \\
\hline Potássio & $3,3 \mathrm{mEq} / \mathrm{L}$ & De 3,5 a 5,0 mEq/L \\
\hline Hemograma completo & \multicolumn{2}{|l|}{} \\
\hline Hemácias & $3,57 \mathrm{milh} . / \mathrm{mm} 3$ & Em mulheres: de 4,00 a 5,40 \\
\hline Hemoglobina & $9,40 \mathrm{~g} / \mathrm{dl}$ & Em mulheres: de 12,00 a 16,00 \\
\hline Hematócrito & $29,3 \%$ & Em mulheres: de 35 a 47 \\
\hline V.C.M & $82 \mathrm{fL}$ & Em mulheres: de 80 a 100 \\
\hline H.C.M & $26 \mathrm{pg}$ & Em mulheres: de 27 a 32 \\
\hline C.H.C.M & $32 \mathrm{~g} / \mathrm{dL}$ & Em mulheres: de 32 a 37 \\
\hline R.D.W & $11,3 \%$ & Em mulheres: até $15 \%$ \\
\hline Série branca & \multicolumn{2}{|l}{} \\
\hline Sem alterações & \multicolumn{2}{|l}{} \\
\hline
\end{tabular}

No terceiro dia de internação na UPA, a paciente teve piora do quadro clínico, com significativo aumento das lesões em alvo atípicas planas e aumento das lesões crostosas em face, pescoço, tronco, membros superiores, abdômen e dorso, queda das sobrancelhas (Figura 2C), aumento da área de destacamento de pele em face e pescoço (Figura 2A). A equipe de saúde constatou também severidade no acometimento da mucosa oral e ocular, com lesões erosivas na língua, lesões crostosas recobertas por pequenos pontos de sangramento nos lábios (Figura 2B) e sinéquia conjuntival em ambos os olhos (Figura 2C). Paciente com quadro clínico de febre com Tax $38,2^{\circ} \mathrm{C}$, hipocorada $(1+/ 4+)$ e hipohidratada, $\mathrm{PA} 120 \times 80 \mathrm{mmHg}, \mathrm{SatO}_{2}: 78 \%$ (macro) e $98 \%$ 


\section{Biomedica Brasiliensia}

(máscara com reservatório); após algumas horas apresentou $\mathrm{SatO}_{2} 68 \%$, justificando a realização de gasometria arterial, que apresentou $\mathrm{SatO}_{2}$ 65\%. A paciente foi submetida a intubação endotraqueal (IOT) e diante da gravidade do caso, necessitou de internação em uma Unidade de Terapia Intensiva (UTI), de onde foi transferida para o Hospital Nossa Senhora da Conceição, também no município de Três Rios/RJ.
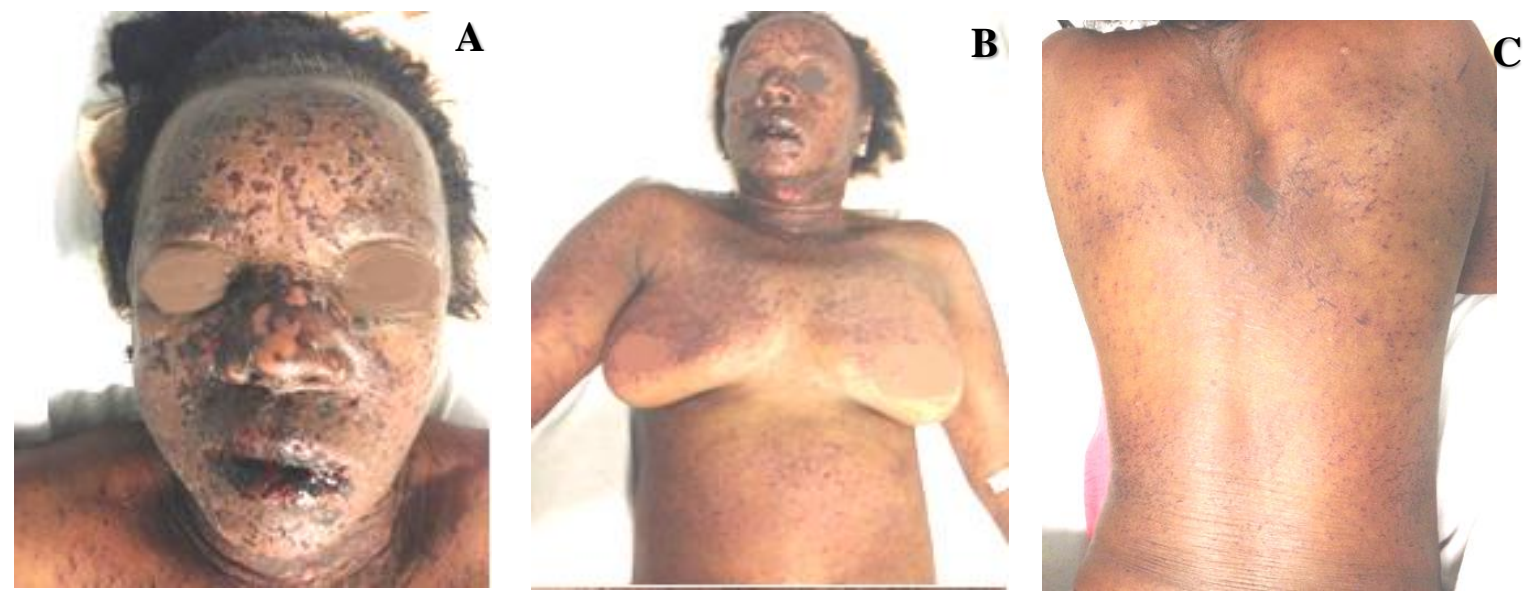

Figura 1: Lesões em alvo atípicas planas e crostosas em (A) face e pescoço, (B) membros superiores e abdômen, (C) dorso.

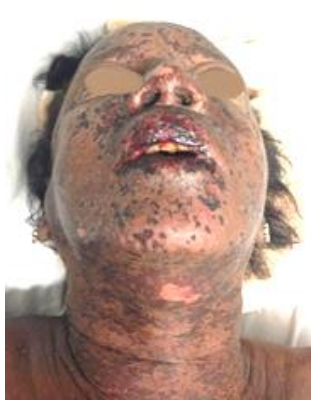

A

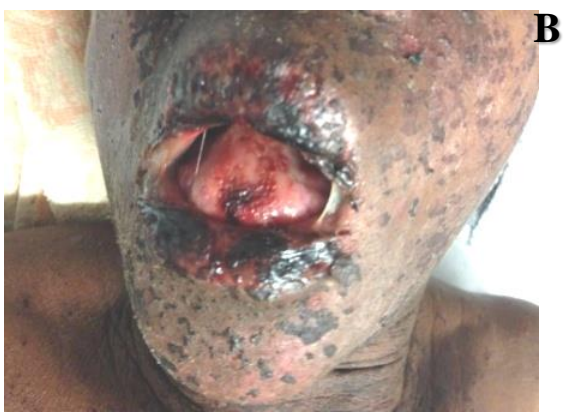

B

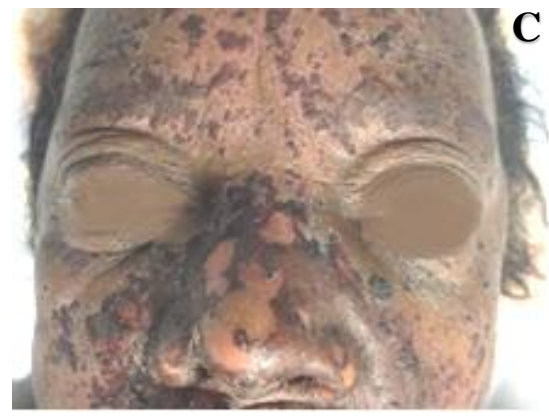

Figura 2: (A) Destacamento de pele em face e pescoço. Atingindo menos de $10 \%$ de destacamento de pele. (B) lesões erosivas na língua, lesões crostosas recobertas por pequenos pontos de sangramento nos lábios. (C)

Acometimento de mucosa ocular e sinéquia conjuntival.

A paciente então deu entrada na UTI do Hospital, hemodinamicamente estável, em IOT e ventilação mecânica, sedada com fentanil $10 \mathrm{ml} / \mathrm{h}$ e midazolan $2 \mathrm{ml} / \mathrm{h}$, com melhora da $\mathrm{SatO}_{2}$ (97\%). Foram solicitados exames de admissão, suporte de UTI/vigilância intensiva e suspenso o uso de prednisona e de anti-hipertensivo.

No segundo dia de internação hospitalar, a paciente evoluiu com edema agudo de pulmão, apresentando ao exame físico MVUA com estertores difusos, PA 190 x $100 \mathrm{mmHg}$, FR 20 irpm, FC 82 bpm e $\mathrm{SatO}_{2}$ 92\%. Foi solicitado radiografia (RX) de tórax que evidenciou sinais de congestão. Para o tratamento do quadro de edema agudo de pulmão, iniciou-se uso do diurético de alça furosemida, $40 \mathrm{mg}$ (2 ampolas) intra venoso (IV) e, do analgésico opióide morfina- 1 ampola em $9 \mathrm{ml}$ de soro fisiológico, administrando $5 \mathrm{mg}$ IV a cada 30 minutos. No período da noite, constatou-se a piora do quadro clínico, com pico febril Tax $38,8^{\circ} \mathrm{C}$. A paciente apresentava-se taquipnéica, FR 42 irpm, PA 170 x $90 \mathrm{mmHg}$, MVUA com creptações bolhosas bilaterais. Foi solicitado então novo RX de tórax, que evidenciou infiltrado intersticial bilateral e opacidade 
organizada em base de hemitórax esquerdo. A dosagem de furosemida foi aumentada para $80 \mathrm{mg}$ + dose de ataque de $20 \mathrm{mg}$. Foi feita reposição de potássio, e iniciado o uso sistêmico do antibiótico cefepima 1 grama de 8 em 8 horas para tratamento dos focos de infecção cutâneas.

No terceiro dia de internação hospitalar, a paciente apresentou grave quadro clínico, com pico febril Tax $40,2^{\circ} \mathrm{C}$, glicemia $256 \mathrm{mg} / \mathrm{dl}$, piora das lesões cutâneas com aumento da área de destacamento de pele em face, pescoço, todo o tronco e abdômen, em membros superiores e por todo o dorso, tendo mais de $30 \%$ do corpo com destacamento de pele. Foi então diagnosticada a evolução do quadro para Necrólise Epidérmica Tóxima (NET). Foi iniciado uso de anticorpos imunoglobulina humana $0,4 \mathrm{~g} / \mathrm{kg} / \mathrm{dia}$ para o tratamento da SSJ $/ \mathrm{NET}$.

Após 5 dias de uso de imunoglobulina humana e com 6 dias de uso de cefepima, portanto, no sétimo dia de internação hospitalar, a paciente apresentou melhora evolutiva significativa das lesões na pele e também melhora do quadro respiratório. Assim, a imunoglobulina humana e a reposição de potássio foram suspensas; cuidados gerais com a pele e cefepima foram mantidos e foi iniciada a redução de sedação e também o uso do anti-hipertensivo anlodipino. No oitavo dia de internação hospitalar, já sem sedação, paciente apresentou abertura ocular aos chamados verbais, atendendo a solicitações simples, interagindo com examinador com o olhar. Encontravase hemodinamicamente estável, taquipnéica sem esforço ventilatório, hipocorada $(1+/ 4+)$, hidratada, acianótica, enchimento capilar satisfatório, MVUA com roncos esparso, PA 140 x 70 mmHg, FC 94 bpm, FR 28 irpm. Foi então solicitado RX de tórax, que não evidenciou sinais de opacidade. A extubação, sem intercorrências, foi realizada e a paciente manteve-se com bom padrão ventilatório, ventilando espontaneamente em macro nebulização com $\mathrm{O}_{2} 5 \mathrm{~L} / \mathrm{min}$.

Após 13 dias de internação hospitalar, a paciente apresentou grande melhora das lesões cutâneas, já sem destacamento de pele e sem acometimento mucoso ocular e oral, apresentandose em bom estado geral, hemodinamicamente estável, atendendo a solicitações, sem déficits motores, com níveis pressóricos estáveis, mantendo-se afebril, com bom padrão respiratório, além de boa oximetria de pulso em ar ambiente. Foi suspenso então o uso de cefepima (em uso há 10 dias) e reduzido o uso de furosemida. A dieta oral pastosa oferecida foi aceita e a paciente não apresentou maiores intercorrências nas 24 horas subsequentes.

No $14^{\circ}$ dia de internação hospitalar, tendo em vista as boas condições clínicas, a paciente foi transferida para enfermaria, onde ficou internada por 2 dias, mantendo-se em bom estado geral, se recuperando das lesões cutâneas que já se encontravam em bom aspecto, sem cicatrizes, não apresentando mais destacamento de pele, com alguns pontos de alteração na pigmentação da pele nos locais das lesões, sem envolvimento de mucosa oral e conjuntival, recebendo alta hospitalar no dia 09/11/2016. Foi orientada a não fazer uso de dipirona, suspeitando-se que tenha sido o medicamento causador da SSJ/NET. Recomendou-se o uso de hidratante corporal em creme no corpo para evitar o ressecamento da pele. A paciente foi encaminhada ao dermatologista e ao oftalmologista para acompanhamento e verificação das sequelas cutâneas e oculares.

\section{Discussão}

O caso aqui de descrito de SSJ-NET apresentou suspeita de ter tido como causa o uso do analgésico dipirona, estando assim em consonância ao citado na literatura científica, que informa que mais de 100 medicamentos podem ser associados ao desencadeamento de SSJ/NET. O analgésico dipirona, nos países em que é comercializada se relaciona frequentemente a essas reações (LUPI et al., 2012; RAMOS E SILVA et al., 2010).

O mecanismo desencadeador inicial da SSJ/NET parece ser mediado por linfócitos citotóxicos, que, por meio das linfocinas IL-6, IL-10 e do fator de necrose tumoral-alfa (TNF- $\alpha$ ) induziriam à apoptose maciça pela ativação dos receptores Fas (glicoproteína transmembrana expressa em várias células). Haveria ainda uma interação com o aumento exagerado na quantidade de óxido nítrico sintetase produzidos nos queratinócitos que, por sua vez, foram estimulados pelo 
aumento do TNF- $\alpha$ e do interferon- $\alpha$, produzidos a partir dos linfócitos T ativados (AZULAY, 2013).

Assim como consta em trabalhos científicos, no caso aqui relatado, o diagnóstico da SSJ/NET foi realizado através de parâmetros clínicos, com o suporte de exames complementares. Clinicamente, a paciente apresentou os sinais típicos e característicos desta síndrome, tais como febre e sintomas não específicos. O quadro cutâneo-mucoso desta alteração é auto limitado e dura em torno de duas a seis semanas (FITZPATRICK, 2011; LUPI et al., 2012). Tanto o acometimento cutâneo como o mucoso possuem intensidade variável, configurando o espectro da síndrome. O quadro da paciente aqui descrito durou aproximadamente cinco semanas, apresentando as lesões comumente citadas em relatos científicos, com máculas eritematosas (lesões em alvo atípicas planas), destacamento de pele inicialmente atingindo menos de $10 \%$ do corpo posteriormente evoluindo para mais de $30 \%$, acometendo face, pescoço, tronco, dorso e membros superiores, além do acometimento de mucosa oral e conjuntival.

Segundo o "Parâmetro SCORTEN de gravidade", desenvolvido por Bastuji-Garin, a paciente descrita nesse caso apresentou 58,3\% de mortalidade prevista, considerando possuir 69 anos de idade, sua porcentagem de superfície corporal desprendida sendo maior que $10 \%$, taxas laboratoriais de ureia de $39,8 \mathrm{mg} / \mathrm{dl}$ e durante sua internação na UTI, ter apresentado pico hiperglicêmico de $256 \mathrm{mg} / \mathrm{dl}$, tento portanto 4 pontos no SCORTEN de gravidade. Isto evidencia a gravidade do caso e prognóstico de mortalidade alto, atendendo ao especificado por diversos autores (AZULAY, 2013; BULISANI et al., 2006; RAMOS E SILVA et al., 2010; FITZPATRICK, 2011).

A paciente apresentou quadro de edema agudo de pulmão enquanto internada na UTI, que segundo a literatura científica está entre as complicações mais frequentes da SSJ/NET e entre as principais causas de mortalidade, que são septicemia por Staphylococcus aureus e Pseudomonas aeruginosa, edema agudo de pulmão, tromboembolismo pulmonar e sangramento gastrintestinal (BULISANI, et al., 2006; EMERICK, et al., 2014; RAMOS E SILVA et al., 2010).

As propostas terapêuticas realizadas atenderam ao preconizado na literatura médica, de modo que o reconhecimento precoce de reação e a retirada do fármaco causador representaram a ação mais importante, visto que o atraso na adoção destas medidas pode ser gravemente prejudicial ao paciente. A literatura preconiza além de cuidados gerais e medidas de suporte para que o paciente sobreviva aos quadros mais graves, assim como o uso de imunoglobulina humana, que se mostra eficaz no tratamento de SSJ/NET, por conter naturalmente anticorpos contra o receptor Fas. Enquanto a antibioticoterapia sistêmica é preconizada em caso de presença de infecção, o uso da corticoterapia sistêmica é controverso na literatura, onde alguns estudos demonstram que essa terapia pode prevenir a progressão da doença quando administrada em fase inicial, o que ocorreu no tratamento da paciente do caso aqui descrito, que fez uso de prednisona $80 \mathrm{mg}$ por 3 dias, onde não foi apresentado eficácia na prevenção da progressão da doença. Outros estudos concluem que os corticóides não impedem que a doença progrida e se associe ao aumento da mortalidade e relacionado a efeito adverso, principalmente uma sepse. Portanto, os corticóides sistêmicos não são recomendados como terapia fundamental na SSJ/NET. Na paciente do caso descrito, logo na fase inicial, suspeitou-se que o fármaco causador fosse a dipirona, sendo assim este foi suspenso imediatamente. Além de cuidados gerais e de medidas de suporte instituidas, a paciente foi tratada em ambiente hospitalar adequado, ficando em isolamento e logo sendo transferida para UTI. Também fez uso de imunoglobulina humana na dose de $0,4 \mathrm{~g} / \mathrm{kg} /$ dia por 5 dias e após apresentar quadro de infecção fez uso de antibioticoterapia com cefepima $1 \mathrm{~g}$ de $8 / 8$ horas por 10 dias, tratamentos esses que demonstraram início a melhora do quadro da paciente (AZULAY, 2013; BULISANI et al., 2006; FITZPATRICK, 2011).

Assim como citado que as sequelas mais comuns são a hiper ou hipopigmentação da pele, as distrofias das unhas e as complicações oculares, isto foi observado na paciente cujo caso aqui se relata, que ao receber alta, apresentava-se com pontos de alteração da pigmentação na pele em 
locais onde havia lesões, porém não apresentava cicatrizes (RAMOS E SILVA et al., 2010; EMERICK, 2014). As lesões dos lábios e da mucosa bucal geralmente se resolvem sem sequelas, porém as lesões oculares são as de maior morbidade, entretanto a paciente evoluiu com melhora de ambas, tanto do acometimento mucoso oral quanto ocular. Mesmo assim sendo encaminhada ao dermatologista e ao oftalmologista para melhor acompanhamento.

Assim como casos descritos na literatura que necessitaram de internação em unidades de terapia intensiva com fluidos intravenosos, medicamentos e suporte nutricional, a paciente demandou internação por um período considerável para a resolutividade do cuidado prestado (FERNÁNDEZ et al., 2011).

\section{Referências}

AZULAY, R.D. Dermatologia. 6. ed. Rio de Janeiro: Guanabara Koogan, 2013.

BULISANI, A.C.P.; SANCHES, G.D.; GUIMARÃES, H.P.; LOPES, R.D.; VENDRAME, L.S.; LOPES ANTONIO, C. Síndrome de Stevens-Johnson e Necrólise Epidérmica Tóxica em Medicina Intensiva. Revista Brasileira de Terapia Intensiva v.18, n.3, p. 292-297, 2006.

CEDEÑO, M.A.; MÁRQUEZ, C.J.E.; TAMAYO, S.J.R. Presentación de una paciente con síndrome de Stevens-Johnson y necrólisis epidérmica tóxica. Correo Científico Médico, v.18, n.4, p.793-799, 2014.

EMERICK, M.F.B.; RODRIGUES, M.M.T.; PEDROSA, D.M.A.S.; NOVAES, M.R.C.G.; GOTTEMS, L.B.D. Síndrome de Stevens-Johnson e Necrólise Epidérmica Tóxica em um hospital do Distrito Federal. Rev. Bras. Enferm., v.67, n.6, p. 898-904, 2014.

FERNÁNDEZ, B.E.J.; MORALES, D. Síndrome de Stevens-Johnson: presentación de un caso. Archivo Médico de Camagüey v.15, n.3, p. 600-609, 2011.

FITZPATRICK, T.B. Tratado de Dermatologia. 7.ed. São Paulo: Revinter, 2011.

LUPI, O.; BELO, J.; CUNHA, P. Rotinas de diagnóstico e tratamento da Sociedade Brasileira de Dermatologia. 2.ed. São Paulo: AC Farmacêutica, 2012.

RAMOS E SILVA, M.; CASTRO, M.C.R. Fundamentos de Dermatologia 2.ed. Rio de Janeiro: Atheneu, 2010.

RIVITTI, E.A. Manual de dermatologia clínica de Sampaio e Rivitti . São Paulo: Artes Médicas, 2014. 